\title{
Successful Heart Transplantation in a Patient who Recovered From COVID-19
}

\author{
Christine Johnstad ${ }^{1}$, David Murray ${ }^{1}$, Ravi Dhingra ${ }^{2}$, Jason Smith $^{2}$, and Amy Fiedler ${ }^{2}$ \\ ${ }^{1}$ University of Wisconsin-Madison \\ ${ }^{2}$ University of Wisconsin
}

November 10, 2020

\begin{abstract}
COVID-19 has created challenges for society and the medical community. While the pandemic continues to unfold, the transplant community has had to pivot to keep recipients, donors, and transplant teams safe given these unprecedented times. This has resulted in a decrease in the number of transplants performed in the United States and an increased number of inactive patients on the UNOS waiting list.1 Waitlist and transplant recipients have an increased risk for acquiring COVID-19. It is speculated that this patient population is particularly vulnerable given their immunocompromised status and the high prevalence of comorbidities.2 Given the uncertainty surrounding the risk of transplant patients contracting COVID-19, there is interest in describing these cases in the literature.
\end{abstract}

\section{Successful Heart Transplantation in a Patient who Recovered From COVID-19}

Christine M. Johnstad, BA ${ }^{1}$., David Murray, MD²., Ravi Dhingra, MD²., Jason W. Smith, MD¹, Amy G. Fiedler, $\mathrm{MD}^{1}$

${ }^{1}$ University of Wisconsin, Madison. Department of Surgery, Division of Cardiothoracic Surgery

${ }^{2}$ University of Wisconsin, Madison. Department of Medicine, Division of Cardiology

Keywords: Heart transplantation, COVID-19

Word Count: 844

Disclosures: None

Funding statement: No funding was obtained for this study

Conflict of interest disclosure: No conflict of interest

Institutional Review Board approval or waiver: No need for IRB approval for this study Patient consent statement: Consent was not obtained and waived

Permission to reproduce material from other sources: Not applicable

Clinical trial registration: Not applicable

Corresponding Author:

Amy G. Fiedler, MD

Assistant Professor of Cardiac Surgery

University of Wisconsin, Madison 
600 Highland Avenue

CSC H4/320

Madison, WI 53792

Phone: (608) 262-3858

Fax: (608)263-0547

Email: fiedler@surgery.wisc.edu

INTRODUCTION:

We present the case of a 63-year-old male who underwent successful orthotopic heart transplantation (OHT) after surviving infection with SARS-CoV-2 (COVID-19). This is the first documented report of a COVID-19 survivor who subsequently underwent successful OHT.

\begin{abstract}
:
COVID-19 has created unprecedented challenges for society, and specifically the medical community. While the pandemic continues to unfold, the transplant community has had to pivot in order to keep recipients, donors, and institutional transplant teams safe given the unique circumstances inherent to solid organ transplantation.

COVID-19 continues to devastate countries and medical systems around the world, at points leading to an inability to continue to provide safe patient care due to systems limitations. As a result, some transplant centers have been forced to decrease their ability to offer transplantation in the midst of the pandemic. This has resulted in an overall decrease in the number of transplants performed in the United States coupled with an increased number of inactive patients on the UNOS waiting list. ${ }^{1}$

Waitlist and transplant recipients have an increased risk for acquiring COVID-19. It is speculated that this patient population is particularly vulnerable and at risk for more severe disease given their immunocompromised status (post-transplant) and the high prevalence of comorbidities (waitlist and post-transplant). ${ }^{2}$ Given the uncertainty surrounding the risk of transplant patients contracting COVID-19 while on the waiting list or post-transplant, there is interest amongst the transplant community to characterize the patients trajectory should they become infected.
\end{abstract}

\title{
CASE PRESENTATION
}

A 63-year-old male was admitted to our institution in May 2020 with shortness of breath and a history of ischemic cardiomyopathy resulting in New York Heart Association Class IV heart failure. In the process of multi-disciplinary workup for consideration for heart transplant, he was found to be COVID-19 positive by PCR assay. The test was repeated and confirmed positive.

He was relocated to the COVID-19 intensive care unit and isolated. Chest radiograph demonstrated a dense left lower lobe pneumonia which was treated with antibiotics. His heart failure was managed with dual inotropes and tailored diuresis.

After two weeks of isolation, he underwent repeat COVID-19 testing which was negative. His respiratory symptoms had improved, and his chest radiography and CT scan showed resolution of the lower lobe infiltrate. As a result, work-up was re-instituted for heart transplant candidacy. Right heart catheterization was obtained which revealed a pulmonary wedge pressure of $33 \mathrm{mmHg}$ and a cardiac index of $1.44 \mathrm{~L} / \mathrm{min} / \mathrm{m} 2$ despite dual inotropic therapy. The decision was made to place an intra-aortic balloon pump. He was listed as UNOS Status 2 for heart transplantation.

A suitable donor organ became available 72 hours following the patient being listed for transplant. He was brought to the operating room and underwent successful OHT. His post-operative course was unremarkable. He was discharged on post-operative day 17 . 


\section{COMMENT:}

The COVID-19 pandemic poses a variety of challenges for transplant programs. Balancing the risk of waitlist mortality, the safety of the transplant team staff, and the unknown outcome of an immunosuppressed recipient contracting COVID-19 is difficult.

To our knowledge, a case of a patient awaiting heart transplant who contracts COVID-19, survives, and then undergoes successful OHT has not been reported in the literature. This case is of interest to demonstrate that OHT is still feasible in a recipient who has negative COVID-19 PCR prior to transplant in the setting of a prior positive test.

As the pandemic evolves, with the inevitable increase in numbers of COVID-19 patients in the population, transplant centers will need to explore the implications of COVID-19 positivity in the pre-transplant and immediate post-transplant period in order to continue to successfully provide heart transplant to our waitlist population.

This is the first patient described in the literature to survive COVID-19 and then undergo successful OHT. Our patient has no obvious sequela of COVID-19 infection and has been COVID negative since time of transplant.

As the global pandemic continues, this case demonstrates the safety and feasibility of continuing to provide OHT to patients who are survivors of COVID-19.

Further study is warranted to elucidate any impact of prior COVID-19 infection on immunosuppressive regimen or possible long standing pulmonary complications post-transplant.

\section{DISCLOSURES}

No acknowledgements. No disclosures. No financial resources were utilized in the creation of this manuscript. No conflict of interest from any author.

\section{REFERENCES}

Hsu, J., Al-Saffar, F., Ardehali, R., et. al, (2020). Heart transplantation in the early phase of the COVID-19 pandemic: A single-center case series. Clinical Transplantation . 2020;00:e14042. doi: 10.1111/ctr.14042

DeFilippis, E., Farr, M., \& Givertz, M. Challenges in Heart Transplantation in the Era of COVID-19. Circulation . 2020;141:2048-2051. doi:10.1161/CIRCULATIONAHA.120.047096 